\title{
Harnessing the secretome of adipose- derived stem cells in the treatment of ischemic heart diseases
}

\author{
Xiaoting $\mathrm{Li}^{2}$, Teng $\mathrm{Ma}^{3}$, Jiacheng $\mathrm{Sun}^{3}$, Mingjing Shen ${ }^{1}$, Xiang Xue ${ }^{1}$, Yongbing $\mathrm{Chen}^{{ }^{* *}}$ and Zhiwei Zhang ${ }^{1 *}$ (D
}

\begin{abstract}
Adipose-derived stem cells (ASCs) are promising therapeutic cells for ischemic heart diseases, due to the ease and efficiency of acquisition, the potential of myocardial lineage differentiation, and the paracrine effects. Recently, many researchers have claimed that the ASC-based myocardial repair is mainly attributed to its paracrine effects, including the anti-apoptosis, pro-angiogenesis, anti-inflammation effects, and the inhibition of fibrosis, rather than the direct differentiation into cardiovascular lineage cells. However, the usage of ASCs comes with the problems of low cardiac retention and survival after transplantation, like other stem cells, which compromises the effectiveness of the therapy. To overcome these drawbacks, researchers have proposed various strategies for improving survival rate and ensuring sustained paracrine secretion. They also investigated the safety and efficacy of phase I and II clinical trials of ASC-based therapy for cardiovascular diseases. In this review, we will discuss the characterization and paracrine effects of ASCs on myocardial repair, followed by the strategies for stimulating the paracrine secretion of ASCs, and finally their clinical usage.
\end{abstract}

Keywords: Adipose-derived stem cells, Ischemic heart diseases, Paracrine effects, Secretome, Myocardial repair, Strategies

\section{Introduction}

Ischemic heart diseases (IHD), specifically acute myocardial infarction (AMI), are still the primary causes of morbidity and mortality globally [1], in spite of the great improvements in medical and surgical therapy. Cardiac transplantation seems to be the last resort available for replacing the terminally exhausted heart. However, the number of donors is limited. Fortunately, stem cellbased therapy provides a promising and burgeoning option for the treatment of impaired myocardial tissue in the twenty-first century. Stem cell transplantation has been well investigated and showed significant effects on cardiac repair, by reducing left ventricle (LV) remodeling and improving cardiac function after MI [2]. The therapeutic stem cells include bone marrow-derived mesenchymal stem cells (BMSCs), adipose-derived stem cells (ASCs), endothelial progenitor cells (EPCs), embryonic stem cells (ESCs), induced pluripotent stem cells (iPS

\footnotetext{
* Correspondence: chentongt@sina.com; marsflyfish@126.com ${ }^{1}$ Department of Cardiothoracic Surgery, The Second Affiliated Hospital of Soochow University, No.1055, Sanxiang Road, Suzhou 215004, China Full list of author information is available at the end of the article
}

cells), and other emerging therapeutic stem cell types for IHD [3]. Nevertheless, ASCs exhibit much more attractive advantages in the treatment of impaired myocardium compared with other stem cell types, owing to the ease and high efficiency of acquisition, the potential of rapid proliferation, and the advantageous properties of myocardial lineage differentiation potential and immune tolerance [4-6].

Numerous experimental and clinical studies have indicated that ASC-based therapy of IHD is surprisingly effective in the restoration of impaired cardiac function [7-9]. However, the exact repair mechanisms still remain controversial. Interestingly, accumulating evidences have shown that the rate of survival and cardiac retention of stem cells is extraordinarily low after transplantation, even though a remarkable improvement of cardiac function has been observed $[7,10]$. Therefore, researchers speculated that the great benefits were predominantly attributed to the paracrine effects rather than the direct differentiation into cardiovascular lineage cells. This hypothesis had been well verified in BMSCs [11, 12] and ASCs [13]. Yang et al. [14] revealed that ASC-derived vascular cells only amount 
to about $9 \%$ of the enhanced angiogenesis after implantation for MI and further confirmed that the paracrine effects of ASCs played a major role underlying cardiac protective function. In fact, the exact paracrine function of ASCs probably depends on the trophic factors secreted by ASCs, including cytokines, growth factors, extracellular microvesicles or exosomes, and micromolecular materials, broadly termed as the secretome [12].

Here, we introduce the current understanding of the ASC secretome and their functional roles in cardiac repair and subsequently discuss in detail the current strategies to stimulate the release of the ASC secretome. Finally, we highlight the clinical application of ASCs for IHD.

\section{Characterization of ASCs and profiling their secretome}

Zuk et al. [15] first found a population of stem cells, which were isolated from human processed lipoaspirate, presented multilineage potential similar to that of bone marrow-derived MSCs and were termed as ASCs later on. BMSCs were first discovered and regarded as the primary source of stromal stem cells for clinical application. Later on, further studies identified that ASCs were even superior to BMSCs because of the ease of isolation, the safety of investigation, and the considerably large amounts available, which became one of the most promising alternatives to BMSCs [16]. Researchers estimated that BMSCs constituted approximately $0.001-0.01 \%$ of the total marrow nucleated cells, whereas the amount of ASCs was much higher than that of BMSCs when isolated from equivalent amounts of adipose and bone marrow tissue [16]. Fraser et al. [6] compared the frequency and yield between ASCs and BMSCs by applying clonogenic assays for either fibroblastoid-like colonies (CFU-F) or colonies expressing alkaline phosphatase (CFU-AP) and found that the frequency of CFU-F and CFU-AP in adipose tissue was 500 -fold more than that in marrow (ASCs: 1 in 100; BMSCs: between $\sim 1$ in 50, 000 and 1 in 100,000). Thus, unlike BM-MSCs, freshly isolated ASCs from adipose tissue can be readily used in therapy without expansion [17].

Like other somatic stem cells, ASCs are characterized by the expression of specific surface immunophenotypes. According to previous reports, the human ASCs express characteristic surface markers including CD9, CD13, CD29, CD44, CD55, CD71, CD90, CD105, and HLA$\mathrm{ABC}$ and negatively express surface markers including CD11b, CD16, CD18, CD31, CD45, CD104, and HLADR [18]. In addition, the phenotype of ASCs mostly resembles bone marrow-derived MSCs, such as CD13, CD29, CD44, CD58, and CD166 [19]. However, the exact expression profile of ASCs remains unclear, due to the inconsistent phenotype seen in the source of different species, the method of acquisition, the stage of cultivation, and the source of adipose tissue depot [19].

Regarding the differentiation potential, ASCs can differentiate into several cell types, including adipose, bone, cartilage, skeletal muscle, cardiac muscle, neuronal cells, hematopoietic cells, endothelial cells, and hepatocytes in the presence of lineage-specific induction factors and specific extracellular milieu [6]. Nevertheless, the differentiation for stromal stem cells might be biased toward the origin tissue [16]. Recent results suggested that ASCs presented a pronounced capacity of adipogenic differentiation compared with BMSCs in vitro [20]. Consistently, researchers [21] demonstrated that BMSCs were more prone to osteogenic differentiation than ASCs in vitro.

Notably, ASCs from different sources displayed distinct characteristics. Ong et al. [22] reported that the surface markers of subcutaneous adipose were significantly different from those of visceral adipose tissue depots. Subcutaneous adipose-derived ASCs showed increased expression of CD10, whereas ASCs from visceral adipose expressed higher levels of CD200. Also, the differentiation potential of ASCs is related to their specific anatomic locations [23]. For instance, the subcutaneous adipose-derived ASCs could differentiate better into mature adipocytes than those from visceral adipose in vitro [24]. Furthermore, Harmelen et al. [25] confirmed that ASCs from the subcutaneous adipose tissue region from obese subjects proliferated faster than those from the omental region. Compared with omental preadipocytes, human abdominal subcutaneous adipose precursors underwent extensively increased lipid accumulation, enhanced adipogenic transcription factor expression, and decreased TNF- $\alpha$-induced apoptosis [26]. Additionally, the ASCs isolated from the superficial adipose layers differentiated quicker to a greater extent than those from the deep adipose layers of abdominoplasty specimens [27]. Except for the adipose tissue depot origin, donor characteristics such as age, sex, and metabolic status also affected the differentiation potential of ASCs [28].

For therapeutic IHD, ASCs display some favorable features, including directly differentiating into cardiovascular lineage cells and offering the secretome such as cytokines, growth factors, and exosomes [29]. A typical ASC secretome profile comprises various growth factors, cytokines, RNAs, and lipid mediators. Several trophic factors secreted by ASCs have been reported, such as vascular endothelial growth factor (VEGF), hepatocyte growth factor (HGF), insulin-like growth factor (IGF)-1, $\beta$-nerve growth factor (NGF), stromal cell-derived factor (SDF) $-1 \alpha$, and exosomes, which are functional in cardiovascular diseases therapy [30]. Through mass spectrometry analysis, Riis et al. [31] revealed that the secretome of human ASCs consisted of 342 proteins in the normoxic condition. They were related to angiogenesis 
and vasculature development, extracellular matrix (ECM) formation, cell adhesion/migration, cell survival/death, and immune regulation.

However, the secretome expression is heterogeneous and diverse in distinct microenvironments and disease status, such as hypoxia, serum-free, obese, and diabetes. Togliatto et al. [32] found that the paracrine factors of obese ASCs, including VEGF, matrix metalloproteinase 2 (MMP-2), and miR-126 in extracellular vesicles, were largely reduced compared with those of non-obese ASCs. Meanwhile, the secretome profile in vivo is much more complicated and dynamic compared with that in vitro. Therefore, the specific and dynamic expression profile of ASCs is a Gordian knot to be unraveled in different microenvironments. The future study should aim at quantifying the secreting factors in the secretome profile of ASCs in vivo.

\section{Functions of the ASC secretome in myocardial repair}

The extensive researches on mechanisms underlying ASC-based therapy for IHD in the past decade have unveiled some of the key functional roles that ASC secretome play in the restoration of impaired cardiomyocytes and improvement of cardiac function [30]. The functions of ASC secretome for cardiac repair are briefly summarized in Fig. 1. And the paracrine factors of ASCs and related pathways are summarized in Fig. 2.

\section{Anti-apoptosis}

After myocardial infarction, a mass of cardiomyocytes undergo the pathological process of apoptosis due to ischemia and hypoxia. Fortunately, using the ASC secretome-based therapy can largely prevent this adverse outcome. Chen et al. [33] showed that the ASCconditioned medium (ASC-CM) could notably mitigate the apoptosis of cardiomyocytes induced by porphyromonas gingivalis endotoxin (Pg-LPS), along with the change of the expression of apoptosis-related factors including t-Bid and caspase-3. Yang et al. [14] also observed that in the MI border zone, the apoptosis of cardiomyocytes was significantly reduced after the transplantation of ASC-CM. Furthermore, through hypoxic induction, the release of VEGF was significantly increased in ASC$\mathrm{CM}$, which exerted the effects of pro-survival and antiapoptosis on endothelial cell [34]. Besides ASC-CM, ASC-derived exosomes (ASC-exo) also show the specific repair function of damaged cardiomyocytes. Exosomes, secreted by most cell types, are lipid bilayer membrane structures with a diameter of about $30-100 \mathrm{~nm}$ [12]. They acted as the carrier to mediate the communication between ASCs and damaged cardiomyocytes by shuttling various micromolecular materials such as proteins and RNAs. Also, ASC-exo could reverse ischemia- or hypoxia-induced myocardial apoptosis. After hypoxia/reoxygenation-induced myocardial injury, ASCexo significantly regulated the expression of apoptosisrelated markers, such as Bax, Bcl-2, and Cyclin D1,

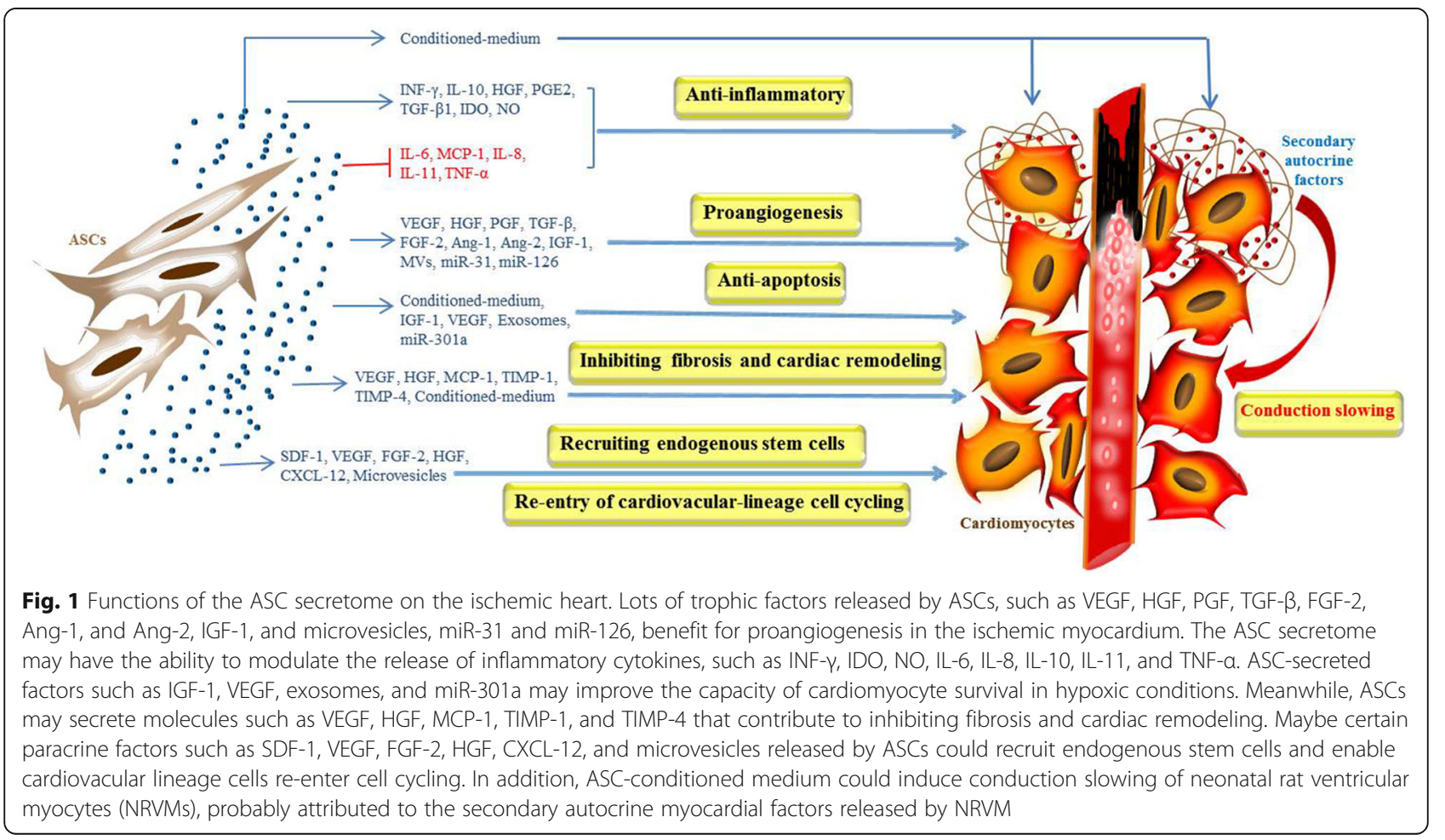




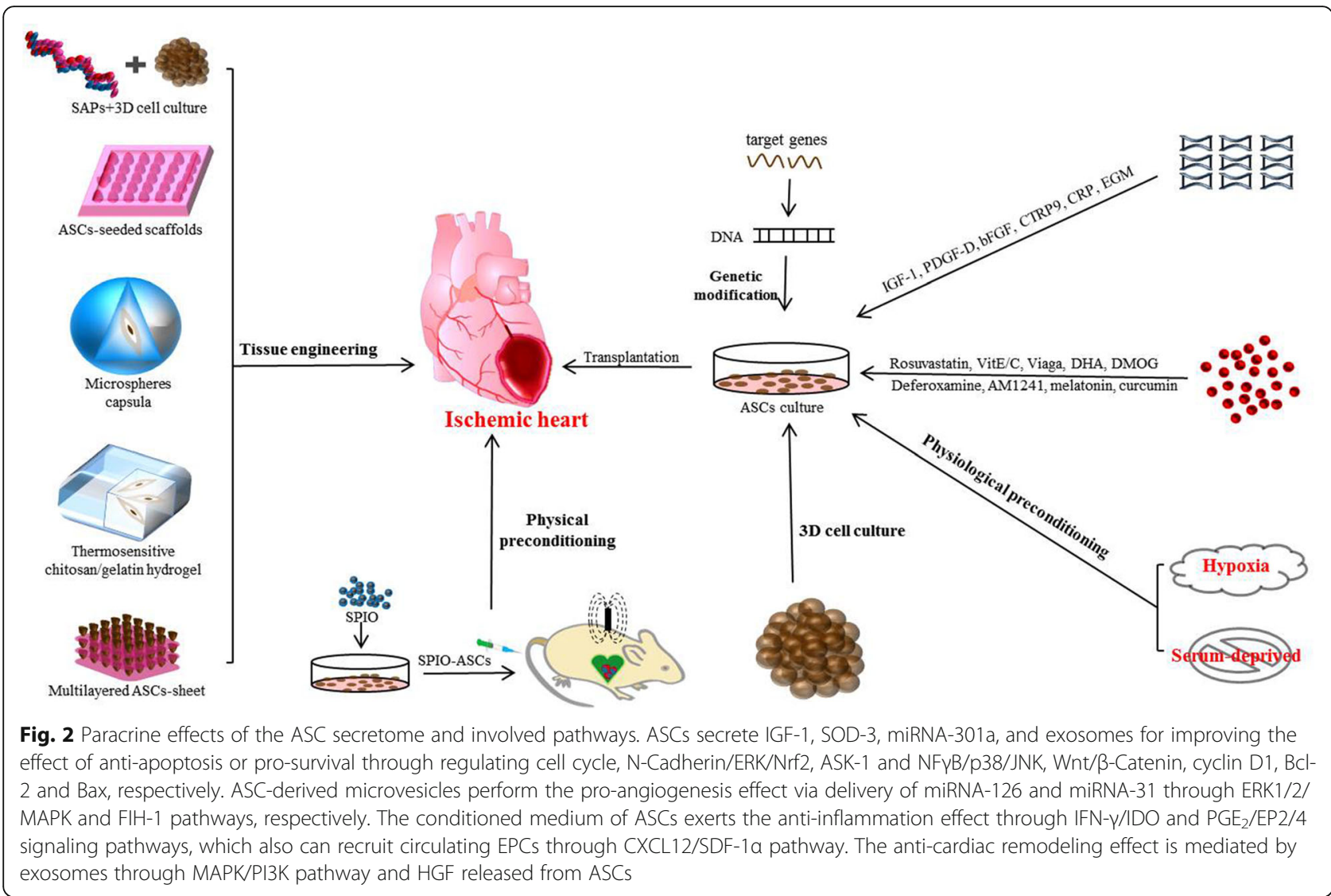

through the Wnt/ $\beta$-catenin signaling pathway [35]. In a variety of delivery RNAs, Lee et al. [36] confirmed that miRNA-301a enriched in human ASCs could significantly attenuate apoptosis of injured cardiomyocytes by inhibiting the apoptosis signal-regulating kinase 1 (ASK1), which regulated the downstream of $\mathrm{p} 38 / \mathrm{JNK} / \mathrm{NF} \gamma \mathrm{B}$ signaling pathways.

\section{Proangiogenesis}

Many researches have reported that the ASC secretome contributes to the angiogenesis in the ischemic heart. Among lots of cytokines, the angiogenesis-related cytokines secreted by ASCs, such as HGF, VEGF, placental growth factor (PGF), transforming growth factor (TGF)$\beta$, fibroblast growth factor (FGF)-2, angiopoietin (Ang)1 , and Ang-2, are able to directly act on endothelial cells and thus increase their pro-angiogenesis capacity [37]. Rehman et al. [34] also observed that the pro-angiogenic potential of ASCs appeared following the secretion of VEGF, HGF, and TGF- $\beta$. Additionally, the microvesicles (MVs) released by ASCs, playing a vital role in intercellular communication, also have the potential of proangiogenesis. Kang et al. [38] delivered microRNA-31 from MVs to endothelial cells (ECs), targeting the factor-inhibiting HIF-1 (FIH-1), and found that MVs could promote the migration and vascular formation of
ECs. Togliatto et al. [32] also found that miRNA-126 in ASC-derived MVs could prompt angiogenesis by activating the extracellular signal-regulated protein kinase $1 / 2 /$ mitogen-activated protein kinase (Erk1/2/MAPK) pathway in patients with diabetes mellitus. Regarding the ASC-based therapy for AMI, Wang et al. [39] demonstrated that the capillary density in the infarct border zone was significantly augmented, resulting from the cardiac protective growth factors secreted by ASCs, such as VEGF, HGF, and IGF-1, although only $0.5 \%$ of the recovered ASCs stained positive for cardiac-specific fibril proteins. In addition, Oliva-Olivera et al. [40] delineated an interesting phenomenon that the level of VEGF in the conditioned medium secreted by ASCs derived from the thymus (thymASCs) was remarkably higher than that from subcutaneous adipose (subASCs). This explains the stronger pro-angiogenic effect of thymASCs than that of subASCs.

\section{Anti-inflammation}

The inflammation is one of the crucial pathophysiological processes after AMI and may lead to poor prognosis. ASCs possess the potential to regulate inflammatory stress through secreting various inflammatory-related factors. Olga et al. [41] illustrated that conditioned supernatants of human ASCs had an obvious impact on the immunization 
of peripheral blood mononuclear cells, accompanied by the release of immunosuppressive soluble factors including interferon (IFN)- $\gamma$, IL-10, HGF, prostaglandin $\mathrm{E}_{2}\left(\mathrm{PGE}_{2}\right)$, TGF- $\beta 1$, indoleamine 2,3-dioxygenase (IDO), and nitric oxide. The IFN- $\gamma / \mathrm{IDO}$ axis might be the essential pathway in the anti-inflammation process. Hao et al. [42] also found that the $\mathrm{PGE}_{2}$ secreted by ASCs were anti-inflammatory, due to the enhanced release of IL-10 by the polarization of activated M2 macrophages through the $\mathrm{PGE}_{2}-\mathrm{EP} 2 / 4$ axis. Regarding the immunoregulation of the ASC secretome on myocardium repair, Kim et al. [43] confirmed that the circulating pro-inflammatory cytokine levels of IL-6 and monocyte chemoattractant protein (MCP)-1 were dramatically decreased after transplantation of mASCs ${ }^{\text {hTERT }}$ (the human telomerase reverse transcriptase gene transfected into mouse ASCs) for AMI therapy, which were remarkably decreased after the application of CD $34^{-} \mathrm{mASCs}{ }^{\text {hTERT }}$. Future studies should put more effort to examine the significant anti-inflammatory effect of ASC secretome in the therapy of IHD.

\section{Inhibition of fibrosis and cardiac remodeling}

MI is frequently accompanied by cardiac fibrosis and remodeling, consequently leading to the development of ventricular dilatation and congestive heart failure. Nevertheless, the transplantation of ASCs provides a unique therapeutic strategy to reverse fibrosis and prevent the impaired heart from adverse matrix remodeling. The remodeling process involves the modulation of various components of ECM and is mediated by the paracrine effects of ASCs. Mazo et al. [44] reported that after the transplantation of ASCs for ischemia/reperfusion-induced swine cardiac injury, the cardiac fibrosis and the ventricular impairment were dramatically improved in 3 months. Although the engraftment of ASCs was not observed, the ratio of MMP/TIMP was changed, which strongly suggested that the paracrine effect may account for the cardiac repair and remodeling. Furthermore, Li et al. [45] had manifested that HGF was a major effective contributor for anti-fibrosis function of ASCs.

Wang et al. [39] also found that the paracrine effect of ASCs may be responsible for the improvement of cardiac function and dilation, as only $0.5 \%$ of the transplanted ASCs were retained in the ASC-treated heart. Furthermore, Yang et al. [14] confirmed that ASC-CM treatment alone, depending on various trophic cardioprotective factors secreted by ASCs in the medium, was sufficient to reduce myocardial infarct size and improve cardiac function for MI mice. To further determine the cardioprotective components in the ASC-CM, they reported that trophic factors, including VEGF, HGF, MCP-1, TIMP-1, and TIMP-4, were beneficial for reducing scar fibrosis and inhibiting cardiac hypertrophy and remodeling for MI therapy [46]. In addition, ASC- derived exosomes could significantly decrease intimal thickness through activation of MAPK/PI3K signaling pathways, contributing to anti-vascular remodeling [47].

\section{Recruitment of endogenous stem cells and re-entry of cardiovascular lineage cell cycling}

Through the paracrine effect, the transplanted ASCs should have the capacity of mobilizing and recruiting autologous stem cells, which reside in the adult tissue or organs such as the peripheral blood, bone marrow, and adipose depot [2, 48]. It was reported that after the transplantation of ASCs, the chemotactic factor of SDF-1 in the blood was increased, and circulating EPCs migrated to the transplanted region and were enriched in the ischemic issue [49]. In addition, in order to improve the efficiency of recruitment, Bhang et al. [50] utilized the technique of three-dimensional (3D) spheroid cultivation to facilitate ASCs releasing much more paracrine factors, such as VEGF, FGF-2, HGF, and chemokine (C-X-C motif) ligand (CXCL)-12. These factors could promote EPC mobilization from the bone marrow and homing to the ischemic location.

Hatzistergos et al. [51] reported that intra-myocardial injection of BMSCs could mobilize host endogenous $\mathrm{c}^{-\mathrm{kit}^{+}}$stem cells, but injection of BMSC-conditioned medium alone could not achieve the same effect. BMSC delivery was likely to provide enormous advantages for the long-standing retention and persistent releasing of paracrine factors from the cells, which served as the basis of cardiac repair. Furthermore, they found that the mitotic cardiomyocytes distinctly increased, indicating the stimulation of endogenous cardiomyocytes and re-entry of cell cycling.

Based on the mechanism of BMSC-based therapy for cardiac repair, we hypothesize that ASCs may also be able to recruit endogenous stem cells to the damaged cardiac region and stimulate cardiomyocyte to re-enter cell cycling through paracrine action. More investigations are still required to further establish the role of paracrine in the functionality of ASCs.

\section{The impact on cardiac electrophysiology}

Although the ASC secretome presents favorable effects in cardiac repair, the potential pro-arrhythmic influence is non-negligible. However, currently, little is known how ASC secretome affects cardiac electrophysiology. It was reported [52] that porcine ASCconditioned medium significantly reduced the conduction velocity of neonatal rat ventricular myocytes (NRVMs) measured by electrical mapping and microelectrode recordings. The secretome of porcine ASCs (pASCs) impaired cardiomyocytes, leading to an increased electrophysiological heterogeneity and conduction dysfunction. Smit et al. [53] further explored 
the underlying mechanism of the potential pro-arrhythmic impact of pASC secretome. They demonstrated that the secretome could induce NRVM to release the secondary autocrine myocardial factors, which were responsible for the cardiac electrophysiological change. Consistently, using a kind of biomaterial, recombinant human collagen-based microspheres, the induced secondary autocrine effect of NRVM was significantly reduced, and finally, the cardiac conduction slowing was notably mitigated. The effects of ASC secretome on cardiac electrophysiology were briefly summarized in Fig. 1.

\section{Strategies for stimulating ASC paracrine secretion}

A major issue of the usage of implanted ASCs in the treatment of IHD is the low cardiac retention rate. Also, the concentration and volume of paracrine factors released by ASCs might be insufficient for restoring the function of an impaired heart. Hence, various strategies have been proposed to increase the survival rate of ASCs and prompt the sustained and regulated paracrine. We summarized those strategies as follows (Fig. 3).

\section{Genetic modification}

The modification of gene expression is a conventional approach to alter the cellular function, including promoting ASCs to release the secretome. Wang et al. [54] found that PHD2 silencing in ASCs, through the transduction with lentiviruses encoding shRNA, notably enhanced the secretion of IGF-1 and helped to reduce cardiomyocyte apoptosis through the activation of NF- $\gamma \mathrm{B}$ binding to IGF-1 gene promoter. Furthermore, to improve the efficiency, Deveza et al. [55] used biodegradable polymeric nanoparticles, poly $\beta$-amino esters, as vectors to transduce the VEGF gene into ASCs. With the increased production of VEGF, ASCs could enhance HUVEC viability, migration, and tube formation. Lee's lab [56] also developed a high-efficiency delivery system that uses poly (ethyleneglycol)-poly (amino ketal) to transfect SDF- $1 \alpha$ into hASCs under the hypoxic condition. This method significantly enhanced the levels of SDF-1a, VEGF, and FGF-2 compared with the conventional approaches. Additionally, the genetic modification of ASCs can further enhance the therapeutic effects by increasing the expression level of desired molecules of the exosomes. For instance, after the overexpression of miR-126 in ASCs, the exosomes were more heavily loaded with miR-126, which can significantly promote microvascular generation and migration, and markedly inhibit cardiac fibrosis and inflammation [57].

\section{Molecular preconditioning of protein}

The trophic proteins prompt ASCs to secrete paracrine factors. For instance, the growth factor, IGF-1, could facilitate ASCs releasing angiogenesis-related factors, such as VEGF-A, TGF- $\beta, \alpha$-smooth muscle actin ( $\alpha$-SMA), FGF-1, MMP-2, and MMP-9 [58]. Another cytokine of platelet-derived growth factor (PDGF)-D also enhanced ASC paracrine activity, leading to the increased release of diverse growth factors including VEGF-A, FGF-1, FGF-5, leukemia inhibitory factor (LIF), and heparin binding EGF-like growth factor, as well as the strengthening of ASCs' regenerative potential [59]. Furthermore, an apoptosis-related protein, $\mathrm{C} 1 \mathrm{q} /$ tumor necrosis factorrelated protein-9, could induce the remarkable augmentation of superoxide dismutase- 3 secretion by ASCs,

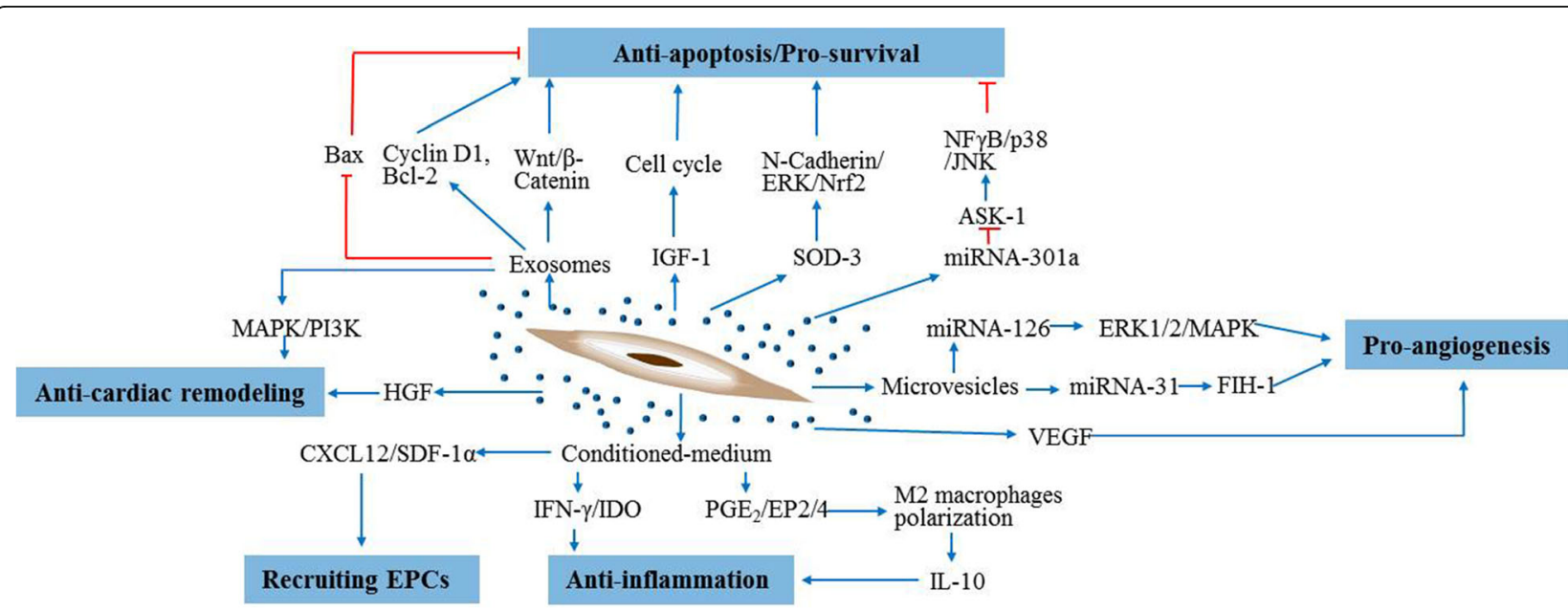

Fig. 3 Strategies for stimulating ASC paracrine secretion are summarized. Genetic modification and protein molecular, pharmacological, physiological, and physical preconditioning have been employed to stimulate the release of the customized ASC secretome. Furthermore, bioengineering strategies, such as 3D cell culture combined with SAPs, ASC-seeded scaffolds, microspheres capsula, thermosensitive chitosan/gelatin hydrogel, and cell sheet, can efficiently control and maintain the release of ASC secretome through several smart biomaterials 
which was involved in the enhanced anti-apoptotic cardio-protection via $\mathrm{N}$-cadherin/ERK/Nrf2 signaling pathway [60]. Specially, a kind of immunization-related protein, C-reactive protein, could promote the upregulation of VEGF-A in ASCs by activating the hypoxia-inducible factor- $1 \alpha$ (HIF-1 $\alpha)$ via CD64/PI3K/ Akt and MAPK/ERK signaling pathways [61]. Kang et al. [38] pretreated ASCs with endothelial differentiation medium, which contained various trophic protein molecules, and found that the release of microvesicles was enhanced, and this in turn contributed to proangiogenesis effect after the delivery of miR-31targeting HIF-1. Consistent with Kang's findings, Souza et al. [62] found that preconditioning of ASCs with endothelial growth medium could stimulate ASCs to secrete HGF and augment their pro-angiogenic function. Additionally, using biomaterial, the controlled release of trophic proteins allowed researchers to stimulate ASCs for a longer period of time. The bFGF retained in the gelatin hydrogel helped to promote ASCs to release HGF, VEGF, and TGF- $\beta 1$ and, thus, contributed to the angiogenesis in the ischemic region [63].

\section{Pharmacological preconditioning}

Various pharmacological molecules, including rosuvastatin [64], vitamin E [65], vitamin C [66], dimethyloxalylglycine [67], docosahexaenoic acid [68], melatonin [69], and curcumin [70], have been documented to enhance the paracrine action of ASCs and are also engaged in the anti-inflammation and anti-oxidative stress, pro-angiogenesis, promoting proliferation, and survival of implanted ASCs. Deferoxamine, an antioxidant compound, could stimulate ASCs to secrete the VEGF in a concentration- and time-dependent manner and thus improved the regenerative function of endothelial cells [71]. Furthermore, deferoxamine also showed a similar effect on diabetic ASCs to restore neovascularization potential through the release of paracrine factors, such as asHIF-1 $\alpha$, VEGF, FGF-2, and SDF-1 [72]. Regarding the treatment for MI, the sildenafil (Viagra, the inhibitor of phosphodiesterase5)-pretreated ASCs could increase the vascular density, decrease the apoptosis and fibrosis of resident cardiomyocytes, and improve cardiac function for MI. These functions are mainly ascribed to the paracrine factors released by activated ASCs such as b-FGF, IGF, and VEGF [73]. Similarly, ASCs preconditioned with AM1241, cannabinoid receptor type II agonist, showed the inhibition of cardiac oxidative stress, apoptosis, and fibrosis. These functions also result from the enhanced release of beneficial factors such as VEGF, bFGF, HGF, and IGF-1, and the decreased level of detrimental factors such as TGF- $\beta 1$ and
PDGF released by ASCs through Stat 3 activation via the phosphorylation of Akt and ERK1/2 [74].

\section{Physiological preconditioning}

Hypoxia or serum-free cultivation has been favored as an effective method to stimulate the release of ASC secretome. Yang et al. [75] confirmed that hypoxia preconditioning of ASCs for $24 \mathrm{~h}$ led to the amplified release of HGF, IL-1, VEGF-A, FGF- 2 , and TGF- $\beta$, contributing to the prevention of apoptosis of cardiomyocytes through the JNK signaling pathway after MI injury. Furthermore, the capillary density in the MI region was also dramatically increased, due to the enhanced secretion of HIF- $1 \alpha$ and VEGF after the treatment of hypoxia (2\% $\mathrm{O}_{2}$ )-preconditioned ASCs [76]. Consistent with Wang's study, Stubbs et al. [77] further elaborated that the increased level of VEGF secreted by hypoxia-preconditioned ASCs was due to the upstream activation of HIF- $1 \alpha$. Interestingly, hypoxic conditioning could also improve the aged ASC secretome, which is in line with the increased level of pro-angiogenic factors such as VEGF, PGF, and HGF, and the decreased level of anti-angiogenic factors such as thrombospondin-1 and plasminogen activator inhibitor (PAI)-1 [78]. The mass spectrometry measurement demonstrated that the most regulated proteomic profiles of hypoxic-preconditioned ASCs were the ECM synthesis and cell metabolism, while several other studies showed that its therapeutic potential was mainly due to the pro-angiogenic effect [31].

In addition, the serum-deprived cultivation could also promote ASCs to change its secretome, including the release of growth factors such asIGF-1, bone morphogenetic protein (BMP)-6, FGF-9, HGF, and PDGF-D; the upregulated expressions of proprotein convertase subtilisin/kexin type (PCSK)-5; and the downregulated expressions of TGF 31 , gremlin (GREM)1, and GREM2. These molecules were responsible for ECM composition and regulating the inflammatory, fibrogenic, and angiogenic pathways [79].

\section{Physical preconditioning}

Physical microenvironmental conditions, such as shear stress, light irradiation, and magnetic and temperature induction, have been identified to control the secretory activity of stem cells [80-84]. Bassaneze et al. [83] reported that the laminar shear stress $\left(10 \mathrm{dyn} / \mathrm{cm}^{2}\right.$ up to $96 \mathrm{~h}$ ) acted on human ASCs could lead to the enhanced secretion of nitric oxide and VEGF. Another physical approach, low-level light irradiation, could also stimulate ASC spheroid to release several angiogenic factors including VEGF and bFGF and to synthesize extracellular matrix such as collagen, contributing to the increased vascular density in the ischemic vascular diseases [80]. Specially, in MI therapy, the transplantation of ASCs 
preloaded with superparamagnetic iron oxide (SPIO) nanoparticles, together with external application of static magnetic field (SMF), could prolong and augment the release of multiple paracrine factors such as VEGF, HGF, and IGF-1 [81]. To boost the retention of ASCs, using another electromagnetic approach, the magnetic cationic liposomes (MCLs) with a positive surface charge could be easily integrated into targeted cell membrane, and thus lead to the accumulation of MCL-labeled ASCs of a high-density level. Additionally, Ishii et al. [84] created a special multilayered ASC sheet for mouse MI therapy. This method led to the enhanced expression of VEGF and bFGF and the increase of myocardial capillaries. Nonetheless, the physical preconditioning methods that present the potential of stimulating ASCs paracrine activity are still limited.

\section{Cell-cell interactions}

3D cell culture can effectively facilitate cell-to-cell interactions and imitate the microenvironment in vivo. These properties poise 3D cell culture as an excellent strategy to promote ASCs to release the secretome. For example, Bhang et al. [85] demonstrated that hASCs cultured as spheroids, especially with a certain critical size over 100-200 mm, exhibited the enhanced secretion of pro-angiogenic and anti-apoptotic factors such as HGF, VEGF, and FGF-2. Furthermore, they found that [47] the injection of hASC-CM from 3D culture could notably improve the blood vessel density and blood perfusion in the hindlimb ischemic diseases compared with the conventional monolayer culture, mostly attributed to the augmented cytokines in 3D culture. Additionally, to further stimulate the release of ASC secretome, Lee et al. [86] combined long-duration 3D culture with hypoxic conditioning, which led to the increase of angiogenic factors (such as VEGF, SDF, and HGF) in a time-dependent manner. Regarding the therapy for MI, transplantation of 3D cell mass (3DCMs) of ASCs could significantly increase the vasculature in the infarcted region, because of the high-level production of VEGF released by 3DCMs [87].

\section{Tissue engineering}

Several biomaterials, such as hydrogels, self-assembling peptides (SAPs), microspheres, cell sheets, and scaffolds, can encapsulate intact cells or be captured into stem cells. This will help to achieve long-term cell retention and engraftment and thus efficiently promotes ASCs to release the secretome.

Various ASC-seeded scaffolds, including extracellular matrix scaffolds and composite scaffolds, could induce the release of angiogenic growth factors. However, the efficiency of ASC paracrine action in different scaffolds was distinct. For instance, the scaffold of small intestinal submucosa preferentially prompted ASCs to release VEGF, compared with the scaffold of a cellular dermal matrix and collagen-chondroitin sulfate-hyaluronic acid [88]. Besides the application of scaffolds, Cheng et al. [89] used a thermosensitive chitosan/gelatin hydrogel to encapsulate hASCs, which led to the enhanced concentration and sustained release of VEGF and the increased capillary density. Furthermore, another type of mixed hydrogel, containing poly (trimethylene carbonate)- $b$ poly (ethylene glycol)- $b$-poly (trimethylene carbonate) diacrylatecopolymers, combined with hypoxia conditioning, presented desirable encapsulation of hASCs and led to a more robust release of angiogenic and chemotactic factors including VEGF-A, angiopoietin-1, angiogenin, HGF, PGF, PDGF-A, leptin, SDF-1 $\alpha$, and MCP-1 [90]. Additionally, in MI therapy, the combination of SAPs and 3D-ASCs culture effectively increased the release of paracrine factors. The VEGF production of the combinational group within the infarcted myocardium was nearly tenfolds and twofolds greater compared with monolayer cell and 3D cell groups, respectively [91]. Another example of comprehensive tissue engineering, combined a facile cell sheet with the VEGF overexpression by genetic engineering, conferred robust and prolonged secretion of growth factors from ASCs, primarily due to the enhanced capacity of retention and survival of implanted ASCs [92].

\section{Application of the ASC secretome in clinical settings}

Various clinical trials for cardiovascular diseases using ASCs have been conducted to demonstrate the safety, feasibility, and efficacy of cell therapy. These results confirm that the cardiac function is improved more or less, probably mediated by the paracrine effect rather than the direct cardiovascular lineage differentiation or myocardial regeneration of ASCs. The applications of ASCs in patients with cardiovascular diseases are summarized in Table 1 (registration at clinicaltrials.gov).

The first clinical trial (NCT00442806) [93] in which autologous ASCs were administered to the patients with AMI was a randomized, double-blinded, parallel assignment, phase I study. This study assessed the safety and feasibility of intracoronary ASC infusion. The main endpoints included the coronary flow, major adverse cardiovascular and cerebrovascular event (MACCE), severe adverse event (SAE), congestive heart failure, LVEF, myocardial infarct size, and perfusion defect during the 6-month follow-up. The results showed that ASC infusion could significantly improve coronary perfusion defect, reduce infarct size, and enhance cardiac function. In addition to intracoronary injection, the PRECISE trial (NCT00426868) [94] reported the safety of trans-endocardial injection of autologous ASCs 


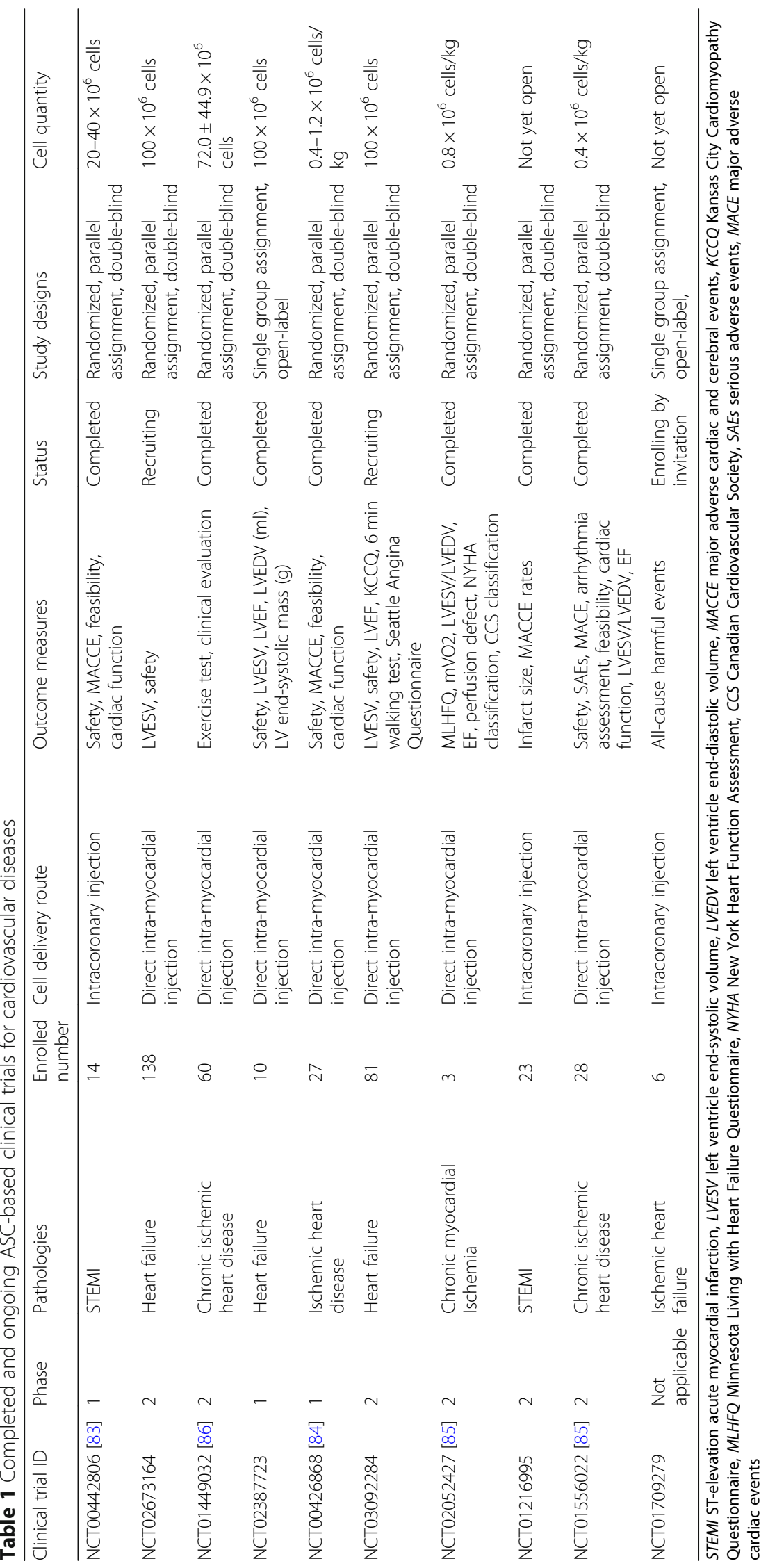


in patients with ischemic cardiomyopathy and displayed favorable effects in cardiac function, myocardial perfusion, and exercise capacity. Except, the ATHENA trials [95] reported another approach to transplant ASCs through direct intra-myocardial injection by electromechanical mapping-guided needle. These studies consisted of two parallel and prospective programs (ATHENA, NCT01556022; ATHENA II, NCT02052427) . Thirty-one patients with an $\mathrm{EF} \geq 20 \%$ but $\leq 45 \%$ (i.e., not eligible for revascularization) and chronic ischemic cardiomyopathy were enrolled. The SPECT results showed a trend in favor of the ASC-treated patients in terms of the differences between ASCs and placebo group for change from baseline in percent LV with stress defect. The total score of the Minnesota Living with Heart Failure Questionnaire (MLHFQ), domain scores for the SF-36, New York Heart Function Assessment (NYHA), and Canadian Cardiovascular Society (CCS) class all showed significant improvement after 12 months of post-implantation. These clinical trials have illustrated the positive efficacy of ASCs. However, the performance of ASC-based treatment did not uniformly meet anticipation. Maybe, different approaches of delivery, cell formulations, and time of ASC administration should affect engraftment efficiency and curative effect. Under the combined actions of these factors, not all output measures were positive or statistically significant in these clinical trials.

Furthermore, the MyStromalCell Trial (NCT01449032) [96] utilized a modified autologous ASCs for the therapy of chronic IHD, that were pretreated with the VEGF-A $\mathrm{A}_{165}$. In this study, the safety of treatment was assessed. Sixty patients with significant coronary artery stenosis, an $\mathrm{EF}>40 \%$, and CCS/NYHA class II-III were enrolled. The results demonstrated that the intra-myocardial

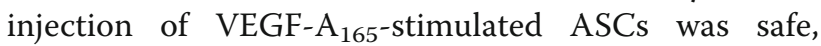
and exercise capacity was significantly increased in the ASC-treated compared to the placebo group. Based on this clinical data, we speculate that the favorable outcomes may be due to the enhancement of secretome released by VEGF- $\mathrm{A}_{165}$-modified ASCs. The ASC modification could promote cell survival and facilitate a sustained and regulated release of paracrine factors.

As for the mechanism of cardiac repair, the paracrine effect is widely considered to be responsible for the improved cardiac function. Nevertheless, it is still difficult to directly demonstrate the involvement of factors in vivo released by ASCs in cardiac functional improvement. Although direct detecting the concentrations of paracrine factors is infeasible in the myocardial tissue in humans, their existence, to a certain extent, could be indirectly indicated by the levels in plasma. Therefore, future clinical trials should endeavor to explore the indicators for ASC-released secretome with favorable sensitivity and specificity. Moreover, it is hard to identify whether paracrine factors originate from transplanted ASCs or host cells, as it is challenging to track these soluble factors. Hence, a comparative analysis of the patient plasma before and after ASC treatment may be useful to provide some information on paracrine effect. In addition, the exact mechanisms of paracrine action have not been fully understood. The analysis of patient plasma could help to reveal the underlying mechanisms in future clinical trials.

\section{Conclusion}

The primary mechanism of the function of ASCs in the therapy for IHD is paracrine. These factors activate local ischemic microenvironment, rescue cardiomyocytes, promote neoangiogenesis, eventually reduce infarct size, and improve cardiac function. However, the therapeutic value of ASC secretome is largely limited without longterm cell retention and engraftment after transplantation. Therefore, various strategies are proposed, including genetic, pharmacological, physiological, physical, and cytokine preconditioning, and tissue engineering, to modulate and stimulate ASCs to release the secretome. Although various effective methods help to prompt the therapeutic value of secretome, the optimal preconditioning condition and the exact triggering and regulatory mechanisms remain elusive, and the functioning proteins and RNAs in the secretome have not yet thoroughly identified. Also, it is still an open question whether the secretome therapy solely is sufficient for cardiac repair. Therefore, further studies should aim to elucidate these pivotal mechanisms and to determine better therapeutic approaches.

\section{Abbreviations}

3D: Three-dimensional; 3DCMs: 3D cell mass; AMI: Acute myocardial infarction; Ang: Angiopoietin; ASCs: Adipose-derived stem cells; ASCCM: ASC-conditioned medium; ASC-exo: ASC-derived exosomes; ASK1: Apoptosis signal-regulating kinase 1; BMP: Bone morphogenetic protein; BMSCs: Bone marrow-derived mesenchymal stem cells; CCS: Canadian Cardiovascular Society; CXCL: Chemokine (C-X-C motif) ligand; ECM: Extracellular matrix; ECs: Endothelial cells; EPCs: Endothelial progenitor cells; ERK1/2/MAPK: Extracellular signal-regulated protein kinase 1/2/mitogen-activated protein kinase; ESCs: Embryonic stem cell; FGF: Fibroblast growth factor; FIH-1: Factor-inhibiting HIF-1; GREM: Gremlin; HGF: Hepatocyte growth factor; HIF-1: Hypoxia-inducible factor-1; IDO: Indoleamine 2,3-dioxygenase; IFN: Interferon; IGF: Insulinlike growth factor; IHD: Ischemic heart diseases; iPS cells: Induced pluripotent stem cells; KCCQ: Kansas City Cardiomyopathy Questionnaire; LIF: Leukemia inhibitory factor; LV: Left ventricle; LVEDV: Left ventricle end-diastolic volume; LVESV: Left ventricle end-systolic volume; MACCE: Major adverse cardiac and cerebral events; MACE: Major adverse cardiac events; MCLs: Magnetic cationic liposomes; MCP: Monocyte chemoattractant protein; MLHFQ: Minnesota Living with Heart Failure Questionnaire; MMP: Matrix metalloproteinase; MVs: Microvesicles; NGF: Nerve growth factor; NRVMs: Neonatal rat ventricular myocytes; NYHA: New York Heart Function Assessment; PAl: Plasminogen activator inhibitor; pASCs: Porcine ASCs; PDGF: Platelet-derived growth factor; $\mathrm{PGE}_{2}$ : Prostaglandin $\mathrm{E}_{2} ;$ PGF: Placental growth factor; SAEs: Serious adverse events; SAPs: Self-assembling peptides; SDF: Stromal cell-derived factor; SMF: Static magnetic field; SPIO: Superparamagnetic iron oxide; STEMI: ST-elevation acute myocardial infarction; subASCs: ASCs derived 
from subcutaneous adipose; TGF: Transforming growth factor; thymASCs: ASCs derived from the thymus; TIMP: Tissue inhibitor of metalloproteinase; VEGF: Vascular endothelial growth factor; a-SMA: aSmooth muscle actin

\section{Acknowledgements}

Not applicable.

\section{Authors' contributions}

$\mathrm{ZZ}$ and $\mathrm{YC}$ were responsible for the concept of the review. XL was responsible for writing the first draft of the manuscript. TM, JS, MS, and XX were responsible for the critical review of the manuscript. All authors read and approved the final manuscript.

\section{Funding}

This study was supported by the National Natural Science Foundation of China (81600217), the Natural Science Foundation of Jiangsu Province (BK20160346), the National Natural Science Foundation of China (81800406), the Suzhou Key Discipline for Medicine (SZXK201803), the Suzhou Science and Technology Foundation (SYS2018064), and the Hospital Internal Research Foundation for PhD (SDFEYBS1709).

\section{Availability of data and materials}

Not applicable.

\section{Ethics approval and consent to participate}

Not applicable.

\section{Consent for publication}

We confirm the table and figures in the manuscript are original for this article.

\section{Competing interests}

The authors declare that they have no competing interests.

\section{Author details}

'Department of Cardiothoracic Surgery, The Second Affiliated Hospital of Soochow University, No.1055, Sanxiang Road, Suzhou 215004, China. ${ }^{2}$ Department of Cardiology, The Second Affiliated Hospital of Soochow University, No.1055, Sanxiang Road, Suzhou 215004, China. ${ }^{3}$ Department of Cardiovascular Surgery, The First Affiliated Hospital \& Institute for Cardiovascular Science, Soochow University, No.899, Pinghai Road, Suzhou 215006, China

Published online: 27 June 2019

\section{References}

1. Benjamin EJ, Blaha MJ, Chiuve SE, et al. Heart disease and stroke statistics2017 update: a report from the American Heart Association. Circulation. 2017;135(10):e146-603.

2. $\mathrm{Yu} \mathrm{H}$, Lu K, Zhu J, Wang J. Stem cell therapy for ischemic heart diseases. Br Med Bull. 2017;121(1):135-54.

3. Jameel MN, Zhang J. Stem cell therapy for ischemic heart disease. Antioxid Redox Signal. 2010;13(12):1879-97.

4. Navarro-Betancourt JR, Baldassarri-Ortego LF, Urquiza-y-Conde F, Hernández S. Adipose tissue-derived stem cells expressing cardiac progenitor markers: the best source of mesenchymal stem cells for cardiovascular repair? Int J Cardiol. 2014;174(2):451-2.

5. Madonna R, Geng Y, De Caterina R. Adipose tissue-derived stem cells. Arterioscler Thromb Vasc Biol. 2009;29(11):1723-9.

6. Fraser JK, Wulur I, Alfonso Z, Hedrick MH. Fat tissue: an underappreciated source of stem cells for biotechnology. Trends Biotechnol. 2006;24(4):150-4.

7. Mazo M, Planat-Bénard V, Abizanda G, et al. Transplantation of adipose derived stromal cells is associated with functional improvement in a rat model of chronic myocardial infarction. Eur J Heart Fail. 2008;10(5):454-62.

8. Naaijkens BA, van Dijk A, Kamp O, Krijnen PAJ, Niessen HWM, Juffermans LJM. Therapeutic application of adipose derived stem cells in acute myocardial infarction: lessons from animal models. Stem Cell Rev Rep. 2014;10(3):389-98.

9. Valina $\mathrm{C}$, Pinkernell $\mathrm{K}$, Song $\mathrm{YH}$, et al. Intracoronary administration of autologous adipose tissue-derived stem cells improves left ventricular function, perfusion, and remodelling after acute myocardial infarction. Eur Heart J. 2007;28(21):2667-77.

10. Hwangbo S, Kim J, Her S, Cho H, Lee J. Therapeutic potential of human adipose stem cells in a rat myocardial infarction model. Yonsei Med J. 2010;51(1):69.

11. Ranganath $\mathrm{SH}$, Levy O, Inamdar MS, Karp JM. Harnessing the mesenchymal stem cell secretome for the treatment of cardiovascular disease. Cell Stem Cell. 2012;10(3):244-58.

12. Sahoo S, Losordo DW. Exosomes and cardiac repair after myocardial infarction. Circ Res. 2014;114(2):333-44.

13. Cai L, Johnstone BH, Cook TG, et al. IFATS collection: human adipose tissuederived stem cells induce angiogenesis and nerve sprouting following myocardial infarction, in conjunction with potent preservation of cardiac function. Stem Cells. 2009;27(1):230-7.

14. Yang $D$, Wang $W$, Li L, et al. The relative contribution of paracrine effect versus direct differentiation on adipose-derived stem cell transplantation mediated cardiac repair. PLoS One. 2013;8(3):e59020.

15. Zuk PA, Zhu M, Mizuno $H$, et al. Multilineage cells from human adipose tissue: implications for cell-based therapies. Tissue Eng. 2001;7(2):211-28.

16. Strioga M, Viswanathan S, Darinskas A, Slaby O, Michalek J. Same or not the same? Comparison of adipose tissue-derived versus bone marrow-derived mesenchymal stem and stromal cells. Stem Cells Dev. 2012;21(14):2724-52.

17. De Ugarte DA, Morizono K, Elbarbary A, et al. Comparison of multi-lineage cells from human adipose tissue and bone marrow. Cells Tissues Organs. 2003;174(3):101-9.

18. Gimble JM, Katz AJ, Bunnell BA. Adipose-derived stem cells for regenerative medicine. Circ Res. 2007;100(9):1249-60.

19. Wankhade UD, Shen M, Kolhe R, Fulzele S. Advances in adipose-derived stem cells isolation, characterization, and application in regenerative tissue engineering. Stem Cells Int. 2016;2016:1-09.

20. Pachon-Pena G, Yu G, Tucker A, et al. Stromal stem cells from adipose tissue and bone marrow of age-matched female donors display distinct immunophenotypic profiles. J Cell Physiol. 2011;226(3):843-51.

21. Noël D, Caton D, Roche $S$, et al. Cell specific differences between human adipose-derived and mesenchymal-stromal cells despite similar differentiation potentials. Exp Cell Res. 2008;314(7):1575-84

22. Ong WK, Tan CS, Chan E, et al. Identification of specific cell-surface markers of adipose-derived stem cells from subcutaneous and visceral fat depots. Stem Cell Reports. 2014;2(2):171-9.

23. Bailey AM, Kapur S, Katz AJ. Characterization of adipose-derived stem cells: an update. Curr Stem Cell Res Ther. 2010;5(2):95-102.

24. Macotela Y, Emanuelli B, Mori MA, et al. Intrinsic differences in adipocyte precursor cells from different white fat depots. Diabetes. 2012;61(7):1691-9.

25. van Harmelen V, Röhrig K, Hauner H. Comparison of proliferation and differentiation capacity of human adipocyte precursor cells from the omental and subcutaneous adipose tissue depot of obese subjects. Metab Clin Exp. 2004;53(5):632-7.

26. Tchkonia T, Tchoukalova YD, Giorgadze N, et al. Abundance of two human preadipocyte subtypes with distinct capacities for replication, adipogenesis, and apoptosis varies among fat depots. Am J Physiol Endocrinol Metab. 2005;288(1):E267-77.

27. Aksu AE, Rubin JP, Dudas JR, Marra KG. Role of gender and anatomical region on induction of osteogenic differentiation of human adipose-derived stem cells. Ann Plast Surg. 2008;60(3):306-22.

28. Cawthorn WP, Scheller EL, MacDougald OA. Adipose tissue stem cells: the great WAT hope. Trends Endocrinol Metab. 2012;23(6):270-7.

29. Planat-Benard V, Silvestre J, Cousin B, et al. Plasticity of human adipose lineage cells toward endothelial cells. Circulation. 2004;109(5):656-63.

30. Ma T, Sun J, Zhao Z, et al. A brief review: adipose-derived stem cells and their therapeutic potential in cardiovascular diseases. Stem Cell Res Ther. 2017;8(1):124.

31. Riis S, Stensballe A, Emmersen J, et al. Mass spectrometry analysis of adipose-derived stem cells reveals a significant effect of hypoxia on pathways regulating extracellular matrix. Stem Cell Res Ther. 2016;7(1):52.

32. Togliatto G, Dentelli P, Gili M, et al. Obesity reduces the pro-angiogenic potential of adipose tissue stem cell-derived extracellular vesicles (EVs) by impairing miR-126 content: impact on clinical applications. Int J Obes. 2016:40(1):102-11.

33. Chen T, Kuo C, Battsengel S, et al. Adipose-derived stem cells decrease cardiomyocyte damage induced by porphyromonas gingivalis endotoxin through suppressing hypertrophy, apoptosis, fibrosis, and MAPK markers. Environ Toxicol. 2018;33(4):508-13. 
34. Rehman J, Traktuev D, Li J, et al. Secretion of angiogenic and antiapoptotic factors by human adipose stromal cells. Circulation. 2004;109(10):1292-8.

35. Cui X, He Z, Liang Z, Chen Z, Wang H, Zhang J. Exosomes from adiposederived mesenchymal stem cells protect the myocardium against ischemia/ reperfusion injury through Wnt/ $\beta$-catenin signaling pathway. J Cardiovasc Pharmacol. 2017;70(4):225-31.

36. Lee C, Shin S, Lee J, et al. MicroRNA-mediated down-regulation of apoptosis signal-regulating kinase 1 (ASK1) attenuates the apoptosis of human mesenchymal stem cells (MSCs) transplanted into infarcted heart. Int J Mo Sci. 2016:17(10):1752.

37. Nakagami H, Maeda K, Morishita R, et al. Novel autologous cell therapy in ischemic limb disease through growth factor secretion by cultured adipose tissue-derived stromal cells. Arterioscler Thromb Vasc Biol. 2005:25(12):2542-7.

38. Kang T, Jones TM, Naddell C, et al. Adipose-derived stem cells induce angiogenesis via microvesicle transport of miRNA-31. Stem Cells Transl Med. 2016;5(4):440-50

39. Wang $L$, Deng J, Tian $W$, et al. Adipose-derived stem cells are an effective cell candidate for treatment of heart failure: an MR imaging study of rat hearts. Am J Phys Heart Circ Phys. 2009;297(3):H1020-31.

40. Oliva-Olivera W, Coín-Aragüez L, Lhamyani S, et al. Differences in the neovascular potential of thymus versus subcutaneous adipose-derived stem cells from patients with myocardial ischaemia. J Tissue Eng Regen Med. 2018;12(3):e1772-84

41. DelaRosa O, Lombardo E, Beraza A, et al. Requirement of IFN-gammamediated indoleamine 2,3-dioxygenase expression in the modulation of lymphocyte proliferation by human adipose-derived stem cells. Tissue Eng Part A. 2009;15(10):2795-806.

42. Hao C, Shintani S, Shimizu Y, et al. Therapeutic angiogenesis by autologous adipose-derived regenerative cells: comparison with bone marrow mononuclear cells. Am J Phys Heart Circ Phys. 2014;307(6):H869-79.

43. Kim J, Choi S, Park C, et al. Transplantation of immortalized CD34+ and CD34- adipose-derived stem cells improve cardiac function and mitigate systemic pro-inflammatory responses. PLoS One. 2016;11(2):e147853.

44. Mazo M, Hernández S, Gavira JJ, et al. Treatment of reperfused ischemia with adipose-derived stem cells in a preclinical swine model of myocardial infarction. Cell Transplant. 2012;21(12):2723-33.

45. Li X, Zhao H, Qi C, Zeng Y, Xu F, Du Y. Direct intercellular communications dominate the interaction between adipose-derived MSCs and myofibroblasts against cardiac fibrosis. Protein Cell. 2015;6(10):735-45.

46. Mazo M, Cemborain A, Gavira JJ, et al. Adipose stromal vascular fraction improves cardiac function in chronic myocardial infarction through differentiation and paracrine activity. Cell Transplant. 2012;21(5):1023-37.

47. Arslan F, Lai RC, Smeets MB, et al. Mesenchymal stem cell-derived exosomes increase ATP levels, decrease oxidative stress and activate PI3K Akt pathway to enhance myocardial viability and prevent adverse remodeling after myocardial ischemia/reperfusion injury. Stem Cell Res. 2013;10(3):301-12.

48. Cesselli D, Beltrami AP, Rigo S, et al. Multipotent progenitor cells are present in human peripheral blood. Circ Res. 2009;104(10):1225-34.

49. Kondo K, Shintani S, Shibata R, et al. Implantation of adipose-derived regenerative cells enhances ischemia-induced angiogenesis. Arterioscler Thromb Vasc Biol. 2009:29(1):61-6.

50. Bhang SH, Lee S, Shin J, Lee T, Jang H, Kim B. Efficacious and clinically relevant conditioned medium of human adipose-derived stem cells for therapeutic angiogenesis. Mol Ther. 2014;22(4):862-72.

51. Hatzistergos $K E$, Quevedo $H$, Oskouei BN, et al. Bone marrow mesenchymal stem cells stimulate cardiac stem cell proliferation and differentiation. Circ Res. 2010;107(7):913-22.

52. Ten Sande JN, Smit NW, Parvizi M, et al. Differential mechanisms of myocardial conduction slowing by adipose tissue-derived stromal cells derived from different species. Stem Cells Transl Med. 2017;6(1):22-30.

53. Smit NW, Ten Sande JN, Parvizi M, et al. Recombinant human collagenbased microspheres mitigate cardiac conduction slowing induced by adipose tissue-derived stromal cells. PLoS One. 2017;12(8):e183481.

54. Wang WE, Yang D, Li L, et al. Prolyl hydroxylase domain protein 2 silencing enhances the survival and paracrine function of transplanted adiposederived stem cells in infarcted myocardium. Circ Res. 2013;113(3):288-300.

55. Deveza L, Choi J, Imanbayev G, Yang F. Paracrine release from nonviral engineered adipose-derived stem cells promotes endothelial cell survival and migration in vitro. Stem Cells Dev. 2013;22(3):483-91.

56. Lee $T$, Shim M, Yu T, et al. Bioreducible polymer micelles based on acid-degradable poly (ethylene glycol)-poly (amino ketal) enhance the stromal cell-derived factor-1agene transfection efficacy and therapeutic angiogenesis of human adipose-derived stem cells. Int Mol Sci. 2018;19(2):529.

57. Luo Q, Guo D, Liu G, Chen G, Hang M, Jin M. Exosomes from miR-126overexpressing ADSCs are therapeutic in relieving acute myocardial ischaemic injury. Cell Physiol Biochem. 2018;44(6):2105-16.

58. Lin S, Zhang Q, Shao X, et al. IGF-1 promotes angiogenesis in endothelial cells/adipose-derived stem cells co-culture system with activation of PI3K Akt signal pathway. Cell Prolif. 2017;50(6):e12390.

59. Hye Kim J, Gyu Park S, Kim W, Song SU, Sung J. Functional regulation of adipose-derived stem cells by PDGF-D. Stem Cells. 2015;33(2):542-56.

60. Yan W, Guo Y, Tao L, et al. C1 q/tumor necrosis factor-related protein-9 regulates the fate of implanted mesenchymal stem cells and mobilizes their protective effects against ischemic heart injury via multiple novel signaling pathways. Circulation. 2017;136(22):2162-77.

61. Chen J, Gu Z, Wu M, et al. C-reactive protein can upregulate VEGF expression to promote ADSC-induced angiogenesis by activating HIF-1a via CD64/PI3K Akt and MAPK/ERK signaling pathways. Stem Cell Res Ther. 2016;7(1):114.

62. Souza LEB, Beckenkamp LR, Sobral LM, et al. Pre-culture in endothelial growth medium enhances the angiogenic properties of adipose-derived stem/stromal cells. Angiogenesis. 2018;21(1):15-22.

63. Horikoshi-Ishihara H, Tobita M, Tajima S, et al. Coadministration of adiposederived stem cells and control-released basic fibroblast growth factor facilitates angiogenesis in a murine ischemic hind limb model. J Vasc Surg. 2016;64(6):1825-34.

64. Zhang Z, Li S, Cui M, et al. Rosuvastatin enhances the therapeutic efficacy of adipose-derived mesenchymal stem cells for myocardial infarction via PI3K Akt and MEKJERK pathways. Basic Res Cardiol. 2013;108(2):333.

65. Bhatti FUR, Kim SJ, Yi A, Hasty KA, Cho H. Cytoprotective role of vitamin E in porcine adipose-tissue-derived mesenchymal stem cells against hydrogenperoxide-induced oxidative stress. Cell Tissue Res. 2018;374(1):111-20.

66. Kim JH, Kim W, Sung YK, et al. The molecular mechanism underlying the proliferating and preconditioning effect of vitamin $C$ on adipose-derived stem cells. Stem Cells Dev. 2014;23(12):1364-76.

67. Chen C, Tang Q, Zhang Y, et al. Metabolic reprogramming by HIF-1 activation enhances survivability of human adipose-derived stem cells in ischaemic microenvironments. Cell Prolif. 2017;50(5):e12363.

68. Parshyna I, Lehmann S, Grahl K, et al. Impact of omega-3 fatty acids on expression of angiogenic cytokines and angiogenesis by adipose-derived stem cells. Atheroscler Suppl. 2017;30:303-10.

69. Zhu P, Liu J, Shi J, et al. Melatonin protects ADSCs from ROS and enhances their therapeutic potency in a rat model of myocardial infarction. J Cell Mol Med. 2015;19(9):2232-43.

70. Liu J, Zhu P, Song P, et al. Pretreatment of adipose derived stem cells with curcumin facilitates myocardial recovery via antiapoptosis and angiogenesis. Stem Cells Int. 2015;2015:1-12.

71. Liu G, Peshavariya HM, Higuchi M, Chan EC, Dusting GJ, Jiang F. Pharmacological priming of adipose-derived stem cells for paracrine VEGF production with deferoxamine. J Tissue Eng Regen Med. 2016;10(3):E167-76.

72. Mehrabani M, Najafi M, Kamarul T, et al. Deferoxamine preconditioning to restore impaired HIF-1alpha-mediated angiogenic mechanisms in adipose-derived stem cells from STZ-induced type 1 diabetic rats. Cell Prolif. 2015;48(5):532-49.

73. Hoke NN, Salloum FN, Kass DA, Das A, Kukreja RC. Preconditioning by phosphodiesterase-5 inhibition improves therapeutic efficacy of adiposederived stem cells following myocardial infarction in mice. Stem Cells. 2012;30(2):326-35.

74. Han D, Li X, Fan W, et al. Activation of cannabinoid receptor type II by AM1241 protects adipose-derived mesenchymal stem cells from oxidative damage and enhances their therapeutic efficacy in myocardial infarction mice via Stat3 activation. Oncotarget. 2017;8(39):64853-66.

75. Yang J, Zhang H, Zhao L, Chen Y, Liu H, Zhang T. Human adipose tissuederived stem cells protect impaired cardiomyocytes from hypoxia/ reoxygenation injury through hypoxia-induced paracrine mechanism. Cell Biochem Funct. 2012;30(6):505-14.

76. Wang J, Xiang B, Deng J, et al. Hypoxia enhances the therapeutic potential of superparamagnetic iron oxide-labeled adipose-derived stem cells for myocardial infarction. J Huazhong Univ Sci Technolog Med Sci. 2017;37(4):516-22.

77. Stubbs SL, Hsiao ST, Peshavariya HM, Lim SY, Dusting GJ, Dilley RJ. Hypoxic preconditioning enhances survival of human adipose-derived 
stem cells and conditions endothelial cells in vitro. Stem Cells Dev. 2012;21(11):1887-96.

78. Efimenko A, Starostina E, Kalinina N, Stolzing A. Angiogenic properties of aged adipose derived mesenchymal stem cells after hypoxic conditioning. J Transl Med. 2011;9(1):10.

79. Tratwal J, Mathiasen AB, Juhl M, Brorsen SK, Kastrup J, Ekblond A. Influence of vascular endothelial growth factor stimulation and serum deprivation on gene activation patterns of human adipose tissue-derived stromal cells. Stem Cell Res Ther. 2015;6(1):62.

80. Park I, Chung P, Ahn JC. Adipose-derived stem cell spheroid treated with low-level light irradiation accelerates spontaneous angiogenesis in mouse model of hindlimb ischemia. Cytotherapy. 2017;19(9):1070-8.

81. Wang J, Xiang B, Deng J, et al. Externally applied static magnetic field enhances cardiac retention and functional benefit of magnetically ironlabeled adipose-derived stem cells in infarcted hearts. Stem Cells Transl Med. 2016;5(10):1380-93.

82. Miyahara Y, Nagaya N, Kataoka M, et al. Monolayered mesenchymal stem cells repair scarred myocardium after myocardial infarction. Nat Med. 2006;12(4):459-65.

83. Bassaneze V, Barauna VG, Lavini-Ramos C, et al. Shear stress induces nitric oxide-mediated vascular endothelial growth factor production in human adipose tissue mesenchymal stem cells. Stem Cells Dev. 2010;19(3):371-8.

84. Ishii M, Shibata R, Shimizu Y, et al. Multilayered adipose-derived regenerative cell sheets created by a novel magnetite tissue engineering method for myocardial infarction. Int J Cardiol. 2014;175(3):545-53.

85. Bhang SH, Cho SW, La WG, et al. Angiogenesis in ischemic tissue produced by spheroid grafting of human adipose-derived stromal cells. Biomaterials. 2011:32(11):2734-47.

86. Lee JH, Han Y, Lee SH. Long-duration three-dimensional spheroid culture promotes angiogenic activities of adipose-derived mesenchymal stem cells. Biomol Ther. 2016;24(3):260-7.

87. Kim JH, Park IS, Park Y, Jung Y, Kim SH, Kim S. Therapeutic angiogenesis of three-dimensionally cultured adipose-derived stem cells in rat infarcted hearts. Cytotherapy. 2013;15(5):542-56.

88. Liu S, Zhang $H$, Zhang $X$, et al. Synergistic angiogenesis promoting effects of extracellular matrix scaffolds and adipose-derived stem cells during wound repair. Tissue Eng Part A. 2011;17(5-6):725-39.

89. Cheng N, Lin W, Ling T, Young T. Sustained release of adipose-derived stem cells by thermosensitive chitosan/gelatin hydrogel for therapeutic angiogenesis. ActaBiomaterialia. 2017;51:258-67.

90. Young SA, Sherman SE, Cooper TT, et al. Mechanically resilient injectable scaffolds for intramuscular stem cell delivery and cytokine release. Biomaterials. 2018;159:146-60.

91. Kim JH, Park Y, Jung Y, Kim SH, Kim S. Combinatorial therapy with threedimensionally cultured adipose-derived stromal cells and self-assembling peptides to enhance angiogenesis and preserve cardiac function in infarcted hearts. J Tissue Eng Regen Med. 2017;11(10):2816-27.

92. Yeh T, Dean Fang Y, Lu C, et al. Baculovirus-transduced, VEGF-expressing adipose-derived stem cell sheet for the treatment of myocardium infarction. Biomaterials. 2014:35(1):174-84

93. Houtgraaf JH, den Dekker WK, van Dalen BM, et al. First experience in humans using adipose tissue-derived regenerative cells in the treatment of patients with ST-segment elevation myocardial infarction. J Am Coll Cardiol. 2012:59(5):539-40.

94. Perin EC, Sanz-Ruiz R, Sánchez PL, et al. Adipose-derived regenerative cells in patients with ischemic cardiomyopathy: the PRECISE trial. Am Heart J. 2014;168(1):88-95.

95. Henry TD, Pepine CJ, Lambert CR, et al. The Athena trials: autologous adipose-derived regenerative cells for refractory chronic myocardial ischemia with left ventricular dysfunction. Catheter Cardiovasc Interv. 2017; 89(2):169-77.

96. Qayyum AA, Mathiasen AB, Mygind ND, et al. Adipose-derived stromal cells for treatment of patients with chronic ischemic heart disease (MyStromalCell trial): a randomized placebo-controlled study. Stem Cells Int. 2017:2017:1-12.

\section{Publisher's Note}

Springer Nature remains neutral with regard to jurisdictional claims in published maps and institutional affiliations.

Ready to submit your research? Choose BMC and benefit from:

- fast, convenient online submission

- thorough peer review by experienced researchers in your field

- rapid publication on acceptance

- support for research data, including large and complex data types

- gold Open Access which fosters wider collaboration and increased citations

- maximum visibility for your research: over $100 \mathrm{M}$ website views per year

At BMC, research is always in progress.

Learn more biomedcentral.com/submissions 